\title{
Model Predictive Control Algorithm Based on Off-Line Region Dependency
}

\author{
Sung Hyun Kim \\ School of Electrical Engineering, University of Ulsan, Daehak-ro 93, Nam-gu, Ulsan 680-749, Republic of Korea \\ Correspondence should be addressed to Sung Hyun Kim; ie222.kim@gmail.com
}

Received 26 April 2016; Accepted 13 June 2016

Academic Editor: Qingsong Xu

Copyright (C) 2016 Sung Hyun Kim. This is an open access article distributed under the Creative Commons Attribution License, which permits unrestricted use, distribution, and reproduction in any medium, provided the original work is properly cited.

\begin{abstract}
This paper presents an efficient MPC algorithm for uncertain time-varying systems with input constraints. The main advantage of this algorithm with respect to other published algorithms is to significantly enlarge the size of the stabilization set without regard to computational burdens. Specially, we introduce an off-line region-dependent MPC scheme to avoid the size limitation of the control horizon caused by huge on-line computational burdens. A numerical example is included to illustrate the validity of the result.
\end{abstract}

\section{Introduction}

Model predictive control (MPC), also known as receding horizon control (RHC), has received much attention in control societies for its ability to simultaneously handle constraints and time-varying behaviors as well as to well track a reference (see [1-3]). The basic concept of MPC is to solve an optimization problem over future time instants at the current time and to use the first one among the solutions as the current control input. Also, the procedure is repeated at each subsequent instant (see $[4,5])$. Over last few years, considerable research has been performed in the area to stabilize systems with physical limits on actuation (see [6-14]). Specification for developing such a constrained MPC algorithm is described well in [13]. Among them, the most intractable requirement is to increase the size of the stabilization set as large as possible.

One of the ways to enlarge the stabilization set is to use a detuned controller designed to obtain a larger invariant target set. However, since the choice of the detuned controller depletes the quality of performance, ones select the method of increasing the finite control horizon $N$ so as to enlarge the stabilization set. The method has $N$ degrees of freedom with which to enlarge the set of feasible initial states since the enlargement of the stabilization set relies on increase in the control horizon $N[10]$. However, ones cannot blindly enlarge the finite control horizon $N$ because of intensification of the on-line computational complexity. Especially for uncertain systems, due to the propagation of uncertainty over the control horizon, the on-line computational complexity grows rapidly [15]. Undoubtedly, the computational explosion can be arrested by not using the free control moves but instead by allowing the state feedback gain $[8,12]$. However, since lack of free control moves may have adverse effects on both the size of the stabilization set and the quality of achievable dynamic performance, it is inevitable to use dual-mode paradigm.

Various methods to reduce computational burdens caused by the propagation of uncertainty over the finite control horizon have been proposed in the literatures $[10,11$, $13,16,17]$. In [16], the difficulty of uncertainty propagation is avoided through the use of state augmentation which introduces a new free variable $c$, which forms the input to a prestabilized loop. In [10,11, 17], recursive state bounding method is used to overcome the intractable computational complexity. Recently, Wan and Kothare have proposed an efficient algorithm which not only dramatically reduces the on-line computation but also significantly enlarges the size of the stabilization set [13]. However, since optimization problems in the literatures remain solved by the on-line computation, the methods still have the restriction on increasing the control horizon $N$. Particularly, the algorithm proposed in [13] fails to produce optimal control moves since a nominal 
system is used instead of an uncertain system to reduce the computational complexity in the process of minimizing the worst-case performance index.

In this paper, we should develop an efficient MPC algorithm which achieves a larger stabilization set of states without regard to computational burdens. To this end, we firstly introduce an off-line region-dependent MPC scheme which overcomes the size limitation of the control horizon caused by huge on-line computational burdens. In the offline procedure, it is impossible to measure beforehand the state at stabilizing open-loop systems, so-called initial state. Thus we should use vertices of an initial state region with the form of hyperboxes instead of the initial state. For this reason, we term the control scheme off-line region-dependent MPC. Next, we should propose two on-line stabilizing MPC which achieve local optimality within the neighborhood of the equilibrium point.

The paper is organized as follows: Section 2 states target systems and assumptions and supplies an on-line constrained robust MPC algorithm with the control horizon $N$. Section 3 supplies an off-line region-dependent MPC algorithm and two on-line MPC algorithms. Section 4 illustrates the performance of the proposed algorithm through an example. Finally, in Section 5, we make some concluding remarks.

\section{System Description}

Consider the following discrete-time uncertain time-varying systems:

$$
x(k+1)=A(k) x(k)+B(k) u(k)
$$

subject to input constraints

$$
-\bar{u} \leq u(k) \leq \bar{u}, \quad \bar{u} \geq 0, \forall k \in[0, \infty),
$$

where $x(k) \in \mathbb{R}^{n}$ denotes the state and $u(k) \in \mathbb{R}^{m}$ denotes the control. Here, it is assumed that the state $x(k)$ is available at each time $k$. Further, throughout this paper, the inequality between vectors means component-wise inequality, and the system matrix $[A(k) B(k)]$ is unknown but belongs to a polytope $\Omega$ at all times $k$. That is,

$$
\begin{aligned}
& {[A(k) B(k)] \in \Omega} \\
& \quad=\operatorname{Co}\left\{\left[\begin{array}{ll}
A_{1} & B_{1}
\end{array}\right],\left[\begin{array}{ll}
A_{2} & B_{2}
\end{array}\right], \ldots,\left[\begin{array}{ll}
A_{L} & B_{L}
\end{array}\right]\right\},
\end{aligned}
$$

where Co denotes the convex hull and $\left[\begin{array}{ll}A_{\ell} & B_{\ell}\end{array}\right]$, for all $\ell=$ $1, \ldots, L$, are vertices of the convex hull. Thus we can see that there exist nonnegative coefficients $\theta_{\ell_{i}} \triangleq \theta_{\ell_{i}}(k+i)$, for all $\ell_{i}=$ $1, \ldots, L$, such that

$$
\left[\begin{array}{ll}
A(k+i) & B(k+i)
\end{array}\right]=\sum_{\ell_{i}=1}^{L} \theta_{\ell_{i}}\left[\begin{array}{ll}
A_{\ell_{i}} & B_{\ell_{i}}
\end{array}\right], \quad \sum_{\ell_{i}=1}^{L} \theta_{\ell_{i}}=1 .
$$

Continuing, to simplify the notation, we define two transition matrices as follows: for $p \geq q$,

$$
\begin{aligned}
& \Phi_{A}(k+p, k+q) \\
& \quad=A(k+p) A(k+p-1) \cdots A(k+q)
\end{aligned}
$$

$$
\begin{gathered}
=\sum_{\ell_{p}=1, \ldots, \ell_{q}=1}^{L \cdots L}\left(\theta_{\ell_{p}} \cdots \theta_{\ell_{q}}\right) A_{\ell_{p}} A_{\ell_{p-1}} \cdots A_{\ell_{q}}, \\
\Phi_{B}(k+p, k+q) \\
=A(k+p) \cdots A(k+q+1) B(k+q) \\
=\sum_{\ell_{p}=1, \ldots, \ell_{q}=1}^{L \cdots L}\left(\theta_{\ell_{p}} \cdots \theta_{\ell_{q}}\right) A_{\ell_{p}} \cdots A_{\ell_{q+1}} B_{\ell_{q}} .
\end{gathered}
$$

In particular, $\Phi_{A}(k+q, k+q)=A(k+q)$ and $\Phi_{B}(k+q, k+q)=$ $B(k+q)$. Then, from $\sum_{\ell_{p}=1, \ldots, \ell_{q}=1}^{L \cdots L} \theta_{\ell_{p}} \cdots \theta_{\ell_{q}}=1$, it follows that

$$
\begin{aligned}
& \Phi_{A}(k+p, k+q) \in \Omega_{A} \\
& \triangleq \operatorname{Co}\left\{\mathscr{A}_{\ell_{q}}^{\ell_{p}}, \forall \ell_{p}, \ldots, \ell_{q}=1, \ldots, L\right\}, \\
& \Phi_{B}(k+p, k+q) \in \Omega_{B} \\
& \triangleq \operatorname{Co}\left\{\mathscr{B}_{\ell_{q}}^{\ell_{p}}, \forall \ell_{p}, \ldots, \ell_{q}=1, \ldots, L\right\},
\end{aligned}
$$

where $\mathscr{A}_{\ell_{q}}^{\ell_{p}} \triangleq A_{\ell_{p}} A_{\ell_{p-1}} \cdots A_{\ell_{q}}$ and $\mathscr{B}_{\ell_{q}}^{\ell_{p}} \triangleq A_{\ell_{p}} \cdots A_{\ell_{q+1}} B_{\ell_{q}}$.

\section{On-Line Constrained Robust MPC}

Consider the following quadratic performance index:

$$
\begin{aligned}
& J_{p, q}(k) \triangleq \sum_{i=p}^{q}\left(x(k+i \mid k)^{T} Q x(k+i \mid k)\right. \\
& \left.+u(k+i \mid k)^{T} R u(k+i \mid k)\right),
\end{aligned}
$$

where $Q>0$ and $R>0$ are given symmetric matrices and $x(k+i \mid k)$ and $u(k+i \mid k)$ denote predicted variables of the state and the input, respectively, with $x(k)=x(k \mid k)$. The online constrained robust model predictive control is aimed at designing a predictive controller that brings system (1) to the steady state and achieving the following robust performance index at each time $k$ :

$$
\begin{aligned}
\min _{u(\cdot \mid k)[A(k+i)} \max _{B(k+i)] \in \Omega, i \geq 0} & J_{0, \infty}(k) \\
\text { subject to } \quad x(k+1+i \mid k) & \\
= & A(k+i) x(k+i \mid k) \\
& +B(k+i) u(k+i \mid k), \\
& \forall i \in[0, \infty), \\
-\bar{u} \leq u(k+i \mid k) \leq \bar{u}, & \forall i \in[0, N-1], \\
-\bar{u} \leq u(k+i \mid k)=F x(k+i \mid k) &
\end{aligned}
$$

$\forall i \in[N, \infty)$. 
Consider a quadratic function $V(i, k)=x(k+i$ । $k)^{T} P x(k+i \mid k), i \geq N$, where $P>0$ is a symmetric matrix. At sampling time $k$, suppose the quadratic function $V(\cdot)$ satisfies the following inequality for all $x(k+i \mid k)$ and $u(k+i \mid k)$ : for $i \geq N$,

$$
\begin{aligned}
& V(i+1, k)-V(i, k) \leq-\left\{x(k+i \mid k)^{T} Q x(k+i \mid k)\right. \\
& \left.\quad+u(k+i \mid k)^{T} R u(k+i \mid k)\right\} .
\end{aligned}
$$

For the robust performance index to be finite, we must have $x(\infty \mid k)=0$, and hence $V(\infty, k)=0$. Summing up (12) from $i=N$ to $i=\infty$, we can obtain

$$
\begin{aligned}
\max _{[A(k+i) B(k+i)] \in \Omega, i \geq N} \quad & J_{N, \infty}(k) \leq V(N, k) \\
& =x(k+N \mid k)^{T} P x(k+N \mid k) .
\end{aligned}
$$

Thus, the min-max optimization problem (8) is given as

$$
\min _{u(\cdot \mid k)} \gamma_{1}+\gamma_{2}
$$

subject to (9), (10), (11), (12),

$$
\begin{array}{ll}
\max _{[A(k+i) B(k+i)] \in \Omega, 0 \leq i \leq N-1} & J_{0, N-1}(k) \leq \gamma_{1}, \\
& x(k+N \mid k)^{T} P x(k+N \mid k) \\
& \leq \gamma_{2} .
\end{array}
$$

Now, based on (9), let us consider

$$
\begin{aligned}
& x_{k}^{k+N-1} \\
& \triangleq\left[\begin{array}{llll}
x(k \mid k)^{T} & x(k+1 \mid k)^{T} & \cdots & x(k+N-1 \mid k)^{T}
\end{array}\right]^{T}, \\
& \mathscr{U}_{k}^{k+N-1} \\
& \triangleq\left[\begin{array}{llll}
u(k \mid k)^{T} & u(k+1 \mid k)^{T} & \cdots & u(k+N-1 \mid k)^{T}
\end{array}\right]^{T}
\end{aligned}
$$

such that

$$
x_{k}^{k+N-1}=\left[\begin{array}{c}
I \\
\Phi_{A}(k, k) \\
\vdots \\
\Phi_{A}(k+N-2, k)
\end{array}\right] x(k \mid k)+\left[\begin{array}{cccc}
0 & 0 & \cdots & 0 \\
\Phi_{B}(k, k) & 0 & \cdots & 0 \\
\vdots & \ddots & \ddots & \vdots \\
\Phi_{B}(k+N-2, k) & \cdots & \Phi_{B}(k+N-2, k+N-2) & 0
\end{array}\right] \mathscr{U}_{k}^{k+N-1} .
$$

Then, with the help of (6), (18) can be rewritten as

$$
\begin{aligned}
& \mathscr{X}_{k}^{k+N-1}=\sum_{\ell_{N-2}=1, \ldots, \ell_{0}=1}^{L \cdots L} \theta_{\ell_{N-2}} \\
& \cdots \theta_{\ell_{0}}\left(\widetilde{\mathscr{A}}_{\ell_{0}}^{\ell_{N-2}} x(k \mid k)+\widetilde{\mathscr{B}}_{\ell_{0}}^{\ell_{N-2}} \mathcal{U}_{k}^{k+N-1}\right),
\end{aligned}
$$

where

$$
\begin{aligned}
& {\left[\widetilde{\mathscr{A}}_{\ell_{0}}^{\ell_{N-2}}\right.} \\
& \left.\quad \widetilde{\mathscr{B}}_{\ell_{0}}^{\ell_{N-2}}\right] \\
& =\left[\begin{array}{c|cccc}
I & 0 & 0 & \cdots & 0 \\
\mathscr{A}_{\ell_{0}}^{\ell_{0}} & \mathscr{B}_{\ell_{0}}^{\ell_{0}} & 0 & \cdots & 0 \\
\vdots & \vdots & \ddots & \ddots & \vdots \\
\mathscr{A}_{\ell_{0}}^{\ell_{N-2}} & \mathscr{B}_{\ell_{0}}^{\ell_{N-2}} & \cdots & \mathscr{B}_{\ell_{N-2}}^{\ell_{N-2}} & 0
\end{array}\right]
\end{aligned}
$$

That is, for $N=1, \widetilde{\mathscr{A}}_{\ell_{0}}^{\ell_{N-2}}=I$ and $\widetilde{\mathscr{B}}_{\ell_{0}}^{\ell_{N-2}}=0$. Thus, based on (17), (15) can be rewritten as

$$
\begin{aligned}
J_{0, N-1}(k)= & \left(X_{k}^{k+N-1}\right)^{T} \widetilde{Q} \mathscr{X}_{k}^{k+N-1} \\
& +\left(\mathcal{U}_{k}^{k+N-1}\right)^{T} \widetilde{R} \mathcal{U}_{k}^{k+N-1} \leq \gamma_{1},
\end{aligned}
$$

where $\widetilde{Q}$ and $\widetilde{R}$ are block-diagonal matrices whose blocks are $Q$ and $R$, respectively. As a result, through Schur complement and by (19), (21) can be transformed into

0

$$
\leq\left[\begin{array}{ccc}
\gamma_{1} & (*) & (*) \\
\widetilde{Q}^{1 / 2} \widetilde{A}_{\ell_{0}}^{\ell_{N-2}} x(k \mid k)+\widetilde{Q}^{1 / 2} \widetilde{\mathscr{B}}_{\ell_{0}}^{\ell_{N-2}} \mathcal{U}_{k}^{k+N-1} & I & (*) \\
\widetilde{R}^{1 / 2} \mathcal{U}_{k}^{k+N-1} & 0 & I
\end{array}\right],
$$

The $N$-step ahead predicted state $x(k+N \mid k)$ is given by

$$
\begin{aligned}
x & (k+N \mid k)=\Phi_{A}(k+N-1, k) x(k \mid k) \\
& +\left[\Phi_{B}(k+N-1, k) \cdots \Phi_{B}(k+N-1, k+N-1)\right] u_{k}^{k+N-1} \\
& =\sum_{\ell_{N-1}=1, \ldots, \ell_{0}=1}^{L \cdots L} \theta_{\ell_{N-1}} \cdots \theta_{\ell_{0}} \\
& \cdot\left(\mathscr{A}_{\ell_{0}}^{\ell_{N-1}} x(k \mid k)+\left[\mathscr{B}_{\ell_{0}}^{\ell_{N-1}} \cdots \mathscr{B}_{\ell_{N-1}}^{\ell_{N-1}}\right] \mathcal{U}_{k}^{k+N-1}\right) .
\end{aligned}
$$


Thus, by analogy to $[8,12]$, conditions (12) and (16) are converted into, respectively,

$$
\begin{aligned}
0 \leq\left[\begin{array}{cccc}
X & (*) & (*) & (*) \\
A_{\ell} X+B_{\ell} Y & X & (*) & (*) \\
Q^{1 / 2} X & 0 & \gamma_{2} I & (*) \\
R^{1 / 2} Y & 0 & 0 & \gamma_{2} I
\end{array}\right], \quad \forall \ell=1, \ldots, L, \\
0 \leq\left[\begin{array}{ccc}
0 & (*) \\
\mathscr{A}_{\ell_{0}}^{\ell_{N-1}} x(k \mid k)+\widehat{\mathscr{B}}_{\ell_{0}}^{\ell_{N-1}} \mathcal{U}_{k}^{k+N-1} & X
\end{array}\right],
\end{aligned}
$$

where $X=\gamma_{2} P^{-1}, Y=F X$, and $\widehat{\mathscr{B}}_{\ell_{0}}^{\ell_{N-1}}=\left[\mathscr{B}_{\ell_{0}}^{\ell_{N-1}} \ldots \mathscr{B}_{\ell_{N-1}}^{\ell_{N-1}}\right]$. At sampling time $k$, suppose that there exist $\gamma_{2}, X>0$, and $Y=F X$ such that (24) and (25) hold. Then, we have

$$
\begin{array}{cl}
\max _{[A(k+j) B(k+j)] \in \Omega, j \geq N} & x(k+i \mid k)^{T} X^{-1} x(k+i \mid k) \\
& <1,
\end{array}
$$

$$
\forall i \geq N+1
$$

Thus, it should be noted that $\mathscr{E} \triangleq\left\{z \in \mathbb{R}^{n} \mid z^{T} X^{-1} z \leq 1\right\}$ becomes an invariant ellipsoidal set for the predicted states $x(k+i \mid k), i \geq N$, of the uncertain system (1). Accordingly, constraint (11) holds if the following condition is satisfied:

$$
\begin{aligned}
0 & \leq\left[\begin{array}{cc}
Z & Y \\
Y^{T} & X
\end{array}\right], \\
Z_{r r} & \leq \bar{u}_{r}^{2}, \quad \forall r=1, \ldots, m,
\end{aligned}
$$

where $\bar{u}_{r}$ is the $r$ th element of $\bar{u}$.

Theorem 1. Let the control input be given by $\mathcal{U}_{k}^{k+N-1}$ and $u(k+$ $i \mid k)=F x(k+i \mid k), i \geq N$. Then, the optimization problem (14) can be solved by the following semidefinite programming:

$$
\min _{X, Y, Z, \mathscr{U}_{k}^{k+N-1}} \gamma_{1}+\gamma_{2}
$$

subject to (10), (22), (24), (25), and (27), where the feedback gain is given by $F=Y X^{-1}$. Here, the proposed MPC algorithm, if initially feasible, robustly asymptotically stabilizes the closedloop system.

Proof. Suppose that the optimization problem has feasible solution $\left(\mathcal{U}_{k}^{k+N-1^{*}}, F^{*}, P^{*}\right)$ at $k$ time instance. Then, at time $k+1$, the following solution is feasible by (24):

$$
\begin{aligned}
& u(k+i+1 \mid k+1)=u^{*}(k+i+1 \mid k), \\
& \forall i=0, \ldots, N-2, \\
& u(k+i+N \mid k+1)=F^{*} x^{*}(k+i+N \mid k),
\end{aligned}
$$

Consider the following quadratic function:

$$
\mathscr{V}(k)=J_{0, N-1}(k)+x(k+N \mid k)^{T} P x(k+N \mid k),
$$

where all weighting matrices are positive definite. Applying (29) to $\mathscr{V}(k+1)$ allows

$$
\begin{aligned}
\mathscr{V}( & +1) \\
= & J_{1, N}^{*}(k) \\
& +x^{*}(k+1+N \mid k+1)^{T} P^{*} x^{*}(k+N+1 \mid k) \\
\leq & J_{1, N-1}^{*}(k)+x^{*}(k+N \mid k)^{T} P^{*} x^{*}(k+N \mid k) \\
< & J_{0, N-1}^{*}(k)+x^{*}(k+N \mid k)^{T} P^{*} x^{*}(k+N \mid k) \\
= & \mathscr{V}^{*}(k), \quad x(k \mid k) \neq 0 .
\end{aligned}
$$

Since $\mathscr{V}(k+1)^{*} \leq \mathscr{V}(k+1)<\mathscr{V}(k)^{*}, \mathscr{V}(k)^{*}$ becomes a Lyapunov function.

\section{Proposed MPC Algorithm}

The $N$-step control moves $\mathcal{U}_{k}^{k+N-1}$ provide degrees of freedom with which to enlarge the set of feasible initial state. However, it is impossible to blindly enlarge the finite control horizon $N$. That is, because the control horizon $N$ is larger, computational burdens for finding the minimizer of minimization (28) are heavier. Namely, we need to consider a limited control horizon $N^{*}$ such that

$$
\begin{array}{ll}
N^{*}=\max & N \\
\text { subject to } & T(N) \leq T_{s},
\end{array}
$$

where $T(N)$ denotes the time required to solve optimization problem (28) for horizon size $N$ and $T_{s}$ denotes the sampling time of (1). Thus, under $N \leq N^{*}$, it is intractable to directly enlarge the stabilization set via the on-line constrained robust MPC strategy. Thus, motivated by the above concern, this paper places major emphasis on developing an efficient MPC algorithm that can enlarge the size of the stabilization set without regard to computational burdens. The proposed algorithm is clearly explained through two procedures.

4.1. Off-Line Procedure. The goal of this section is to propose an off-line region-dependent MPC algorithm to enlarge the size of the allowable set for initial states, regardless of the horizon limit $N \leq N^{*}$. To this end, we first assume that the initial state of (1) can be measured in advance. Then, we can obtain the $N$-step control moves $\mathscr{U}_{0}^{N-1}$ and the decision variables $X_{0}=\left.X\right|_{k=0}, Y_{0}=\left.Y\right|_{k=0}$, and $Z_{0}=\left.Z\right|_{k=0}$ from the off-line computation of (28). Here, the $N$-step control moves $\mathcal{U}_{0}^{N-1}$ steer the initial state, which lies outside the invariant ellipsoid target set $\mathscr{E}_{0} \triangleq\left\{z \in \mathbb{R}^{n} \mid z^{T} X_{0}^{-1} z \leq 1\right\}$, into the set $\mathscr{E}_{0}$. That is, although the sequence $\mathscr{U}_{0}^{N-1}$ is not optimal on stabilizing (1) from time $k=1$ to $k=N-1$, we can arrange the 


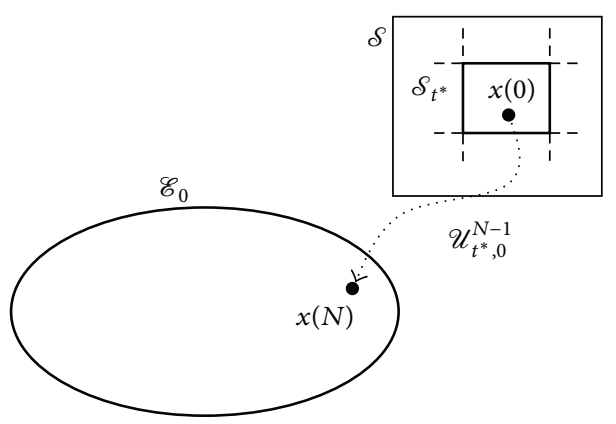

FIGURE 1: $\mathcal{S}_{t^{*}}$ : the divided region including the initial state $x(0), \mathcal{S}$ : the initial state region, and $\mathscr{E}_{0}$ : an invariant ellipsoidal target set.

$N$-step control moves without regard to the limited control horizon $N^{*}$. However, different from the above assumption, it may be hard to measure the initial state $x(0)$ in advance. Thus, as an alternative, this paper considers an initial state region $\mathcal{S}$ subject to $x_{0}^{\min } \leq x(0) \leq x_{0}^{\max }$ instead of $x(0)$, where $x_{0}^{\min }$ and $x_{0}^{\max }$ are assumed to be able to be taken roughly. That is, the region $\delta$ has the form of $n$-dimensional hyperboxes with $2^{n}$ corners. However, in this case, if the initial state region $\delta$ is too extensive, the obtained result is likely to be conservative. Thus, to alleviate such concerns, this paper provides a method of finely dividing $\mathcal{S}$ into some smaller regions $\mathcal{S}_{t}, t=1, \ldots, M$, subject to

$$
\mathcal{S}_{t} \triangleq \operatorname{Co}\left\{x_{t, i}, \quad i=1, \ldots, 2^{n}\right\}
$$

where $x_{t, i}$ are known vertices of the convex hull (see Figure 1).

The following theorem provides a method of designing the control inputs $\mathscr{U}_{t, 0}^{N-1}$ for each region $\mathcal{S}_{t}, t=1, \ldots, M$.

Theorem 2. Given the known vertices $x_{t, i}, i=1, \ldots, 2^{n}$, the initial state included in the set $\mathcal{S}_{t}$ is steered into the invariant ellipsoid target set $\mathscr{E}_{0}$ if there exist symmetric positive definite matrices $X_{0}$ and $Y_{0}$ and matrix $Z_{0}$ and control sequence $\mathcal{U}_{t, 0}^{N-1}$ that are solutions of the following optimization problem:

$$
\min _{X_{0}, Y_{0}, Z_{0}, U_{t, 0}^{N-1}} \gamma_{1}+\gamma_{2}
$$

subject to (10), (24), and (27), for $i=1, \ldots, 2^{n}$,

$$
\begin{aligned}
& 0 \leq\left[\begin{array}{ccc}
\gamma_{1} & (*) & (*) \\
\widetilde{Q}^{1 / 2} \widetilde{\mathscr{A}}_{\ell_{0}}^{\ell_{N-2}} x_{t, i}+\widetilde{Q}^{1 / 2} \widetilde{\mathscr{B}}_{\ell_{0}}^{\ell_{N-2}} \mathcal{U}_{t, 0}^{N-1} & I & (*) \\
\widetilde{R}^{1 / 2} \mathcal{U}_{t, 0}^{N-1} & 0 & I
\end{array}\right], \\
& \forall 1 \leq \ell_{0}, \ldots, \ell_{N-2} \leq L \\
& 0 \leq\left[\begin{array}{cc}
1 & (*) \\
\mathscr{A}_{\ell_{0}}^{\ell_{N-1}} x_{t, i}+\widehat{\mathscr{B}}_{\ell_{0}}^{\ell_{N-1}} \mathcal{U}_{t, 0}^{N-1} & X_{0}
\end{array}\right], \\
& \forall 1 \leq \ell_{0}, \ldots, \ell_{N-1} \leq L
\end{aligned}
$$

Proof. Suppose that the initial state $x(0)$ is included in the set $\delta_{t^{*}}$. Then, by (33), there exist $2^{n}$ nonnegative coefficients $\theta_{t, i}$ such that

$$
\begin{aligned}
& \sum_{i=1}^{2^{n}} \theta_{t, i}=1, \\
& x(0)=\sum_{i=1}^{2^{n}} \theta_{t, i} x_{t, i} .
\end{aligned}
$$

We observe that (35) are affine in the vertices $x_{t, i}$. By multiplying (35) by $\theta_{t, i}$ and, then, by summing them up for $i=1, \ldots, 2^{n}$, we obtain

$$
\begin{gathered}
0 \leq\left[\begin{array}{cccc}
\gamma_{1} & (*) & (*) \\
\widetilde{Q}^{1 / 2} \widetilde{\mathscr{A}}_{\ell_{0}}^{\ell_{N-2}} x(0)+\widetilde{Q}^{1 / 2} \widetilde{\mathscr{B}}_{\ell_{0}}^{\ell_{N-2}} \mathcal{U}_{t, 0}^{N-1} & I & (*) \\
\widetilde{R}^{1 / 2} \mathcal{U}_{t, 0}^{N-1} & 0 & I
\end{array}\right], \\
0 \leq\left[\begin{array}{cc}
\quad & (*) \\
1 & X_{0}, \ldots, \ell_{N-2} \leq L, \\
\mathscr{A}_{\ell_{0}}^{\ell_{N-1}} x(0)+\widehat{\mathscr{B}}_{\ell_{0}}^{\ell_{N-1}} \mathcal{U}_{t, 0}^{N-1} & \\
& \forall 1 \leq \ell_{0}, \ldots, \ell_{N-1} \leq L .
\end{array}\right.
\end{gathered}
$$

Since optimization (28) for the initial state $x(0)$ is recovered, the initial state $x(0)$ in the set $\mathcal{S}_{t}$ is steered into the invariant ellipsoid target set $\mathscr{E}_{0}$ through the $N$-step control moves $\mathcal{U}_{t, 0}^{N-1}$ obtained by the optimization problem (34).

Algorithm 3. Assume that the optimization problem (34) is feasible for all $t=1, \ldots, M$.

(i) Store the $N$-step control moves $\mathcal{U}_{t, 0}^{N-1}$ for each region in a look-up table.

(ii) Search the region $\mathcal{S}_{t^{*}}$ including the initial state $x(0)$ among the separated regions.

(iii) Apply the $N$-step control moves that correspond to the region $\mathcal{S}_{t^{*}}$ to system (1).

4.2. On-Line Procedure. To achieve local optimality within the neighborhood of the equilibrium, we adopt the paradigm 
used in [13]. To realize the paradigm, we must firstly solve two optimization problems:

(i) Obtain $\gamma_{2}^{*}, X^{*}$, and $Y^{*}$ by minimizing $\gamma_{2}$ subject to (24) and $X \geq \lambda^{2} I$ for any $\lambda$.

(ii) Obtain $\left(\gamma_{f}, X_{f}, Y_{f}, Z_{f}\right)$ by minimizing $\beta$ subject to $X<X_{0},(24),(27)$, and $X \geq \lambda^{2} I$ with $\gamma_{2}=\beta \gamma_{2}^{*}, X=$ $\beta X^{*}, Y=\beta Y^{*}$ for the largest possible $\lambda$.

Here, the local control gain is $F_{f}=Y_{f} X_{f}^{-1}=Y^{*}\left(X^{*}\right)^{-1}$.

4.2.1. For $N^{*} \geq 1$. To converge the state inside the invariant ellipsoid $\mathscr{E}_{0}$ to the equilibrium point, let us consider the following optimization problem with the limited control horizon $N^{*}$ :

$$
\min _{X, Y, Z, U_{k}^{k+N^{*}-1}} \gamma_{1}+\gamma_{2}
$$

subject to (24),

0

$$
\begin{aligned}
& \leq\left[\begin{array}{ccc}
\gamma_{1} & (*) & (*) \\
\widetilde{Q}^{1 / 2} \widetilde{A}_{\ell_{0}}^{\ell^{*}-2} x(k \mid k)+\widetilde{Q}^{1 / 2} \widetilde{\mathscr{B}}_{\ell_{0}}^{\ell^{*}-2} \mathcal{U}_{k}^{k+N^{*}-1} & I & 0 \\
\widetilde{R}^{1 / 2} \mathcal{U}_{k}^{k+N^{*}-1} & 0 & I
\end{array}\right], \\
& 0 \leq\left[\begin{array}{rr}
1 & (*) \\
\mathscr{A}_{\ell_{0}^{\ell^{*}-1}}^{\ell^{*}} x(k \mid k)+\widehat{\mathscr{B}}_{\ell_{0}}^{\ell_{N^{*}-1}} \mathcal{U}_{k}^{k+N^{*}-1} & X
\end{array}\right], \\
& 0 \leq\left[\begin{array}{cc}
Z & Y \\
Y^{T} & X
\end{array}\right], \quad \forall 1 \leq \ell_{N^{*}-2}, \ldots, \ell_{N^{*}-1} \leq L, \\
& Z_{r r} \leq \bar{u}_{r}^{2}, \quad \forall r=1, \ldots, m, \\
& -\bar{u} \leq u(k+i \mid k) \leq \bar{u}, \quad \forall i \in\left[0, N^{*}-1\right] .
\end{aligned}
$$

After obtaining, from (38), $N^{*}$-step control moves $\mathcal{U}_{k}^{k+N^{*}-1}$ and a control gain $F$ applied after the $N^{*}$-steps, we implement only the first one as the current control law. This control scheme has the stability property identical with Theorem 1.

Algorithm 4. Suppose that the state of system (1) lies already in the invariant ellipsoid set $\mathscr{E}_{0}$ through the control moves $\mathcal{U}_{t, 0}^{N-1}$. Given $x(N \mid N)$, solve, by on-line computation, optimization problem (38) subject to (24) and (39). And then apply $u(N \mid N)$ to system (1). At any time $k \geq N+1$, let $x(k \mid k)$ be the state.

(i) If the predicted state $x\left(k+N^{*}-1 \mid k-1\right)$ lies outside $\mathscr{E}_{f}=\left\{z \in \mathbb{R}^{n} \mid z^{T} X_{f}^{-1} z \leq 1\right\}$, solve (38) subject to (24) and (39). Apply $u(k \mid k)$.

(ii) If the predicted state $x\left(k+N^{*}-1 \mid k-1\right) \in \mathscr{E}_{f}$ and $N^{*} \geq 2$, redefine $N^{*}=N^{*}-1$ and solve (38) subject to (24) and (39). Apply $u(k \mid k)$. (iii) If the predicted state $x\left(k+N^{*}-1 \mid k-1\right) \in \mathscr{E}_{f}$ and $0 \leq N^{*} \leq 1$, apply $u(k)=F_{f} x(k)$ ever after, where $F_{f}=Y_{f} X_{f}^{-1}$. Set $N^{*}=0$.

Remark 5. In Algorithm 4, we need to check whether the $N^{*}$ step ahead predicted state $x\left(k+N^{*}-1 \mid k-1\right)$, for $k \geq$ $N+1$, lies inside the set $\mathscr{E}_{f}$ or not. To this end, we consider the following numerical criterion:

$$
\begin{aligned}
& \left(\mathscr{A}_{\ell_{0}}^{\ell_{N^{*}-1}} x(k-1)+\widehat{\mathscr{B}}_{\ell_{0}}^{\ell_{N^{*}-1}} \mathcal{U}_{k-1}^{k+N^{*}-2}\right)^{T} \\
& \quad \cdot X_{f}^{-1}\left(\mathscr{A}_{\ell_{0}}^{\ell_{N^{*}-1}} x(k-1)+\widehat{\mathscr{B}}_{\ell_{0}}^{\ell^{*}-1} \mathcal{U}_{k-1}^{k+N^{*}-2}\right) \leq 1 .
\end{aligned}
$$

If it holds, then the $N^{*}$-step ahead predicted state $x\left(k+N^{*}-\right.$ $1 \mid k-1) \in \mathscr{E}_{f}$ for $k \geq N+1$.

Theorem 6. Suppose that the off-line optimization problem (34) is feasible for all $t=1, \ldots, M$. Then the control scheme in Algorithm 4 robustly asymptotically stabilizes the closed-loop system.

Proof. By solving the optimization problem (34) for all $t=$ $1, \ldots, M$, we can construct a look-up table with the $N$-step control moves $\mathscr{U}_{t, 0}, t=1, \ldots, M$. Using the $N$-step control moves $\mathcal{U}_{t^{*}, 0}^{N-1}$ for the region $\mathcal{S}_{t^{*}}$, we include the state $x(N \mid N)$ in the set $\mathscr{E}_{0}$. Here, since the set $\mathscr{E}_{0}$ is an invariant ellipsoidal set, the following solution is feasible for optimization (38) at time $k=N: u(N+i \mid N)=F_{0} x(N+i \mid N), \forall i \geq 0$, where the control gain $F_{0}=Y_{0} X_{0}^{-1}$. Since optimization (38) is feasible initially at time $k=N$, the $N^{*}$-step ahead predicted state $x\left(k+N^{*} \mid k\right)$ is steered into the set $\mathscr{E}_{f}$ through the control moves $\mathscr{U}_{k}^{k+N^{*}-1}$. At any time $k \geq N+1$, suppose $x(k-1+$ $\left.N^{*} \mid k-1\right) \in \mathscr{E}_{f}$ and $N^{*} \geq 2$. Then, after the first control $u(k-1)$ of $\mathcal{U}_{k-1}^{k+N^{*}-2}$ is applied, the remaining $N^{*}-1$ control moves provide a feasible solution for optimization (38) with the control horizon $N^{*}-1$ at time $k$. In case that $x(k \mid k-1) \in$ $\mathscr{E}_{f}$ and $N^{*}=1$ at any time $k \geq N+1$, the control $u(k-1)=$ $\mathscr{U}_{k-1}^{k-1}$ steers the state $x(k \mid k)$ into the set $\mathscr{E}_{f}$. After that, we use continuously the local controller $u(k)=F_{f} x(k)$ to keep the state inside $\mathscr{E}_{f}$ as well as to converge it to the origin.

4.2.2. For $N^{*}=0$. In this case, we use the algorithm identical with the control scheme presented already in [13].

Remark 7. To sum up, this paper develops an off-line algorithm capable of practically implementing the MPC law without regard to the limited control horizon. That is, the efficiency of the proposed algorithm can be clarified from the viewpoint of balancing the tradeoffs between the performance enhancement and the computational complexity.

\section{Numerical Examples}

All optimization problems are solved by using the Matlab LMI-Toolbox (see [18]) on a PC with a Pentium IV processor (speed $1.6 \mathrm{GHz}$, Cache RAM $256 \mathrm{kB}$, and total memory $512 \mathrm{MB})$. 


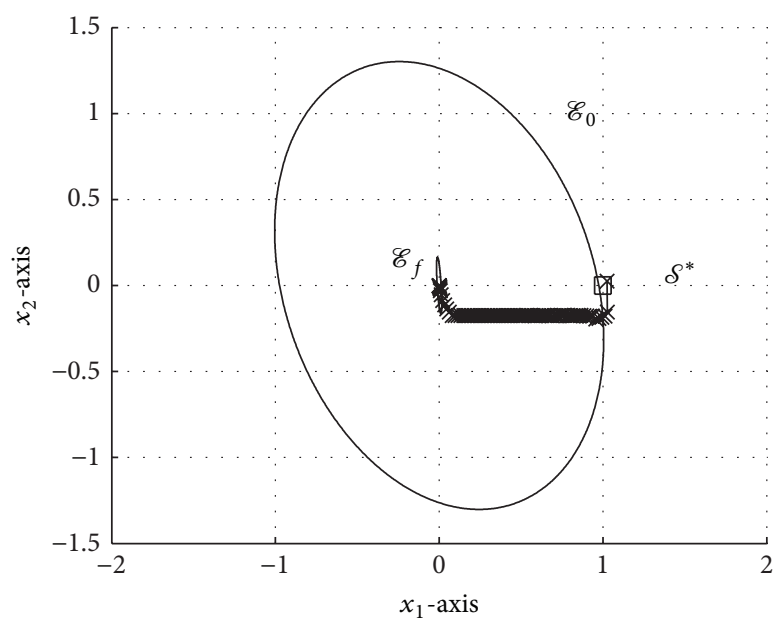

(a)

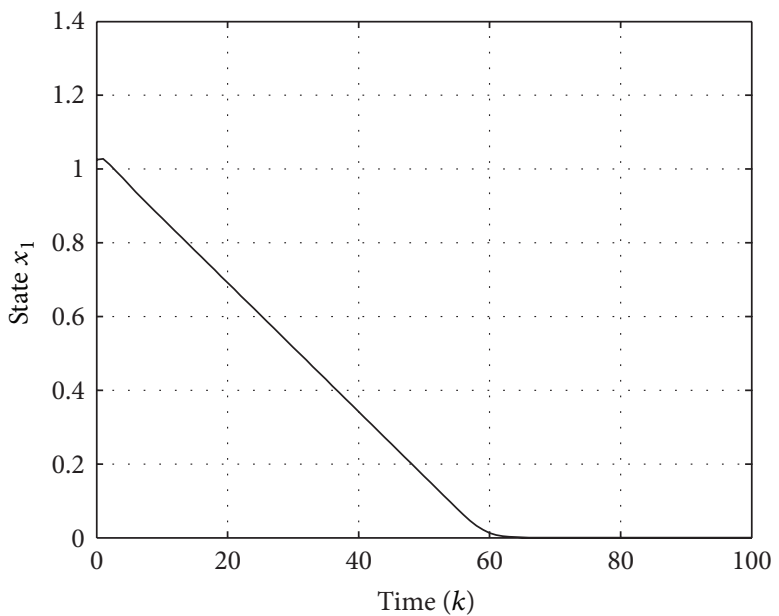

(c)

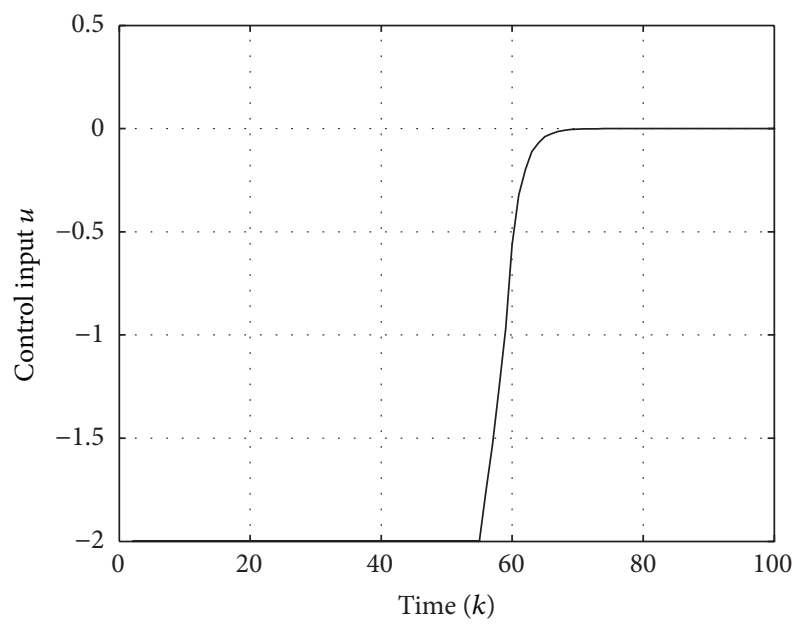

(b)

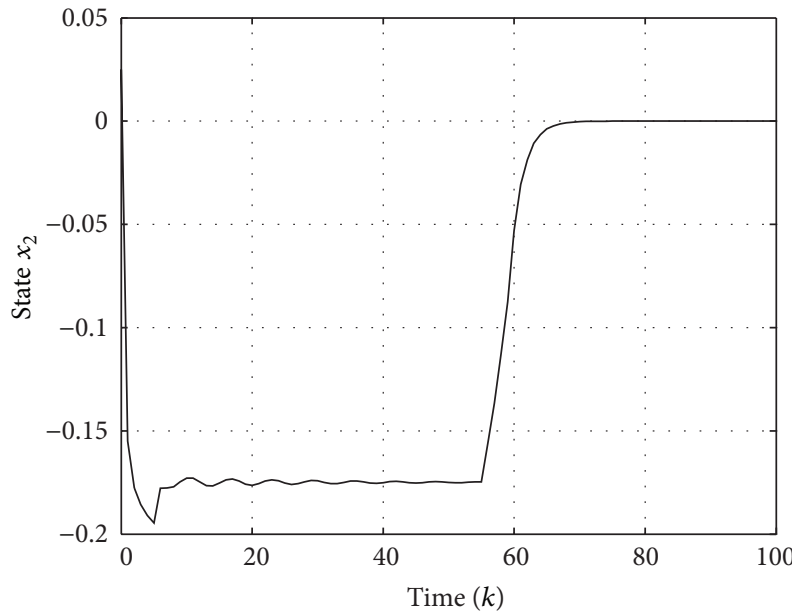

(d)

Figure 2: Results by using the algorithm with the initial state $x(0)=\left[\begin{array}{ll}1.025 & 0.025\end{array}\right]^{T}$ and $\alpha(k)=-2 e^{-0.05 k} \cos (k)$.

Example 1. In order to test the effectiveness of the robust MPC technique described, we revisit Example 1 reported in [8]. The system consists of a classical angular positioning system whose discrete-time equivalent, obtained using a sampling time of $0.1 \mathrm{~s}$ and Euler's first-order approximation of the derivative, is

$$
\begin{array}{r}
{[A(k) \mid B(k)]=\left[\begin{array}{cc|c}
1 & 0.1 & 0 \\
0 & 1-0.1 \cdot \alpha(k) & 0.0787
\end{array}\right],} \\
0.1 \leq \alpha(k) \leq 10 .
\end{array}
$$

From the variation of $\alpha(k)$, we have $A(k) \in \Omega=\operatorname{Co}\left\{A_{1}, A_{2}\right\}$, where

$$
\left[A_{1} \mid A_{2}\right]=\left[\begin{array}{cc|cc}
1 & 0.1 & 1 & 0.1 \\
0 & 0.99 & 0 & 0
\end{array}\right] .
$$

A control constraint of $|u(k)| \leq 2$ is imposed and $J_{0, \infty}(k)$ is given by (7) with

$$
\begin{aligned}
Q & =\left[\begin{array}{ll}
1 & 0 \\
0 & 0
\end{array}\right], \\
R & =0.00002 .
\end{aligned}
$$

We can select an arbitrary initial control horizon $N$ without regard to the sampling time: $N=5$. And then, from investigation of average time to solve optimization (38), we can know that the limited control horizon $N^{*}=2$. Figure 2 shows various simulation results for the proposed algorithm: (a) the invariant ellipsoidal target sets, $\mathscr{E}_{0}$ and $\mathscr{E}_{f}$, and the hyperbox region $\mathcal{S}^{*}$ including the initial state $x(0)$ and (b)-(d) closed-loop responses. As shown in Figure 2, the proposed algorithm is useful for enlarging the stabilization set.

Example 2. To test the effectiveness of the robust MPC algorithm described, we consider the two-mass-spring system 


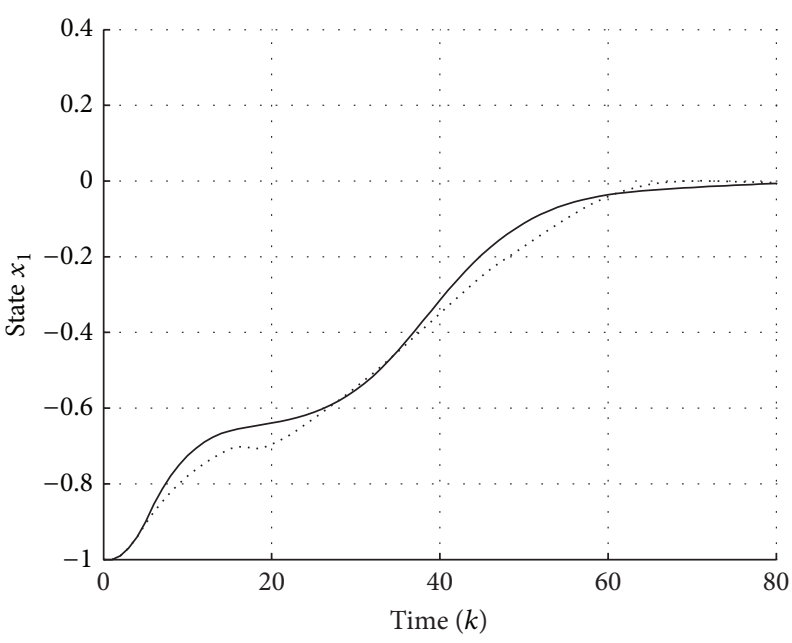

(a)

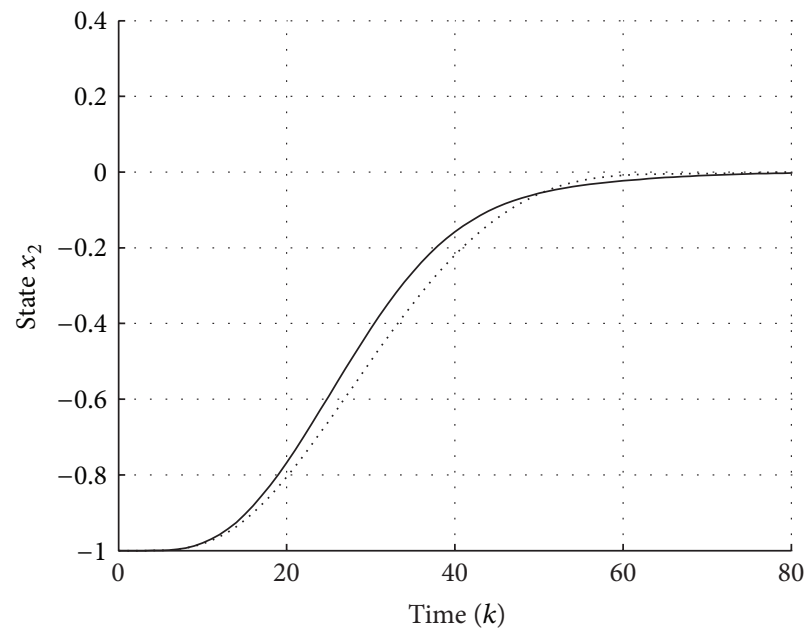

(b)

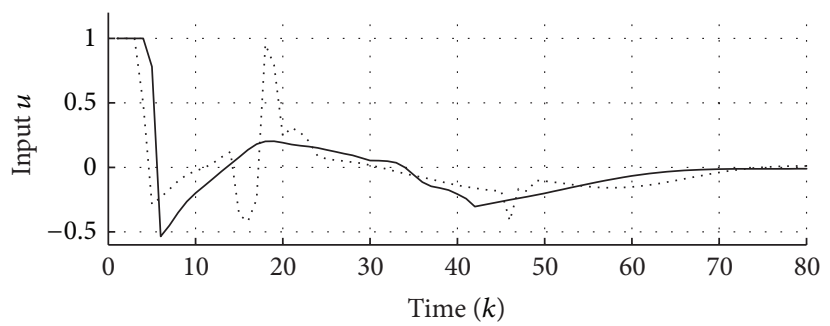

(c)

Figure 3: Comparison between the algorithm [13] with $N=2$ (dotted line) and the proposed algorithm with $N=5$ and $N^{*}=1$ (solid line): (a)-(b) state response and (c) control inputs.

reported in [13]. Its discrete-time equivalent is obtained using a sampling time of $0.1 \mathrm{~s}$ and Euler's first-order approximation of the derivative:

$$
\begin{aligned}
& {[A(k) \mid B(k)]} \\
& \quad=\left[\begin{array}{cccc|c}
1 & 0 & 0.1 & 0 & 0 \\
0 & 1 & 0 & 0.1 & 0 \\
-0.1 \cdot \beta(k) & 0.1 \cdot \beta(k) & 1 & 0 & 0.1 \\
0.1 \cdot \beta(k) & -0.1 \cdot \beta(k) & 0 & 1 & 0
\end{array}\right], \\
&
\end{aligned}
$$

From the variation of $\beta(k)$, we have $A(k) \in \Omega=\operatorname{Co}\left\{A_{1}, A_{2}\right\}$, where

$$
\begin{aligned}
& {\left[A_{1} \mid A_{2}\right]} \\
& =\left[\begin{array}{cccc|cccc}
1 & 0 & 0.1 & 0 & 1 & 0 & 0.1 & 0 \\
0 & 1 & 0 & 0.1 & 0 & 1 & 0 & 0.1 \\
-0.05 & 0.05 & 1 & 0 & -0.12 & 0.12 & 1 & 0 \\
0.05 & -0.05 & 0 & 1 & 0.12 & -0.12 & 0 & 1
\end{array}\right]
\end{aligned}
$$

A control constraint of $|u(k)| \leq 1$ is imposed and the controller design parameters $Q=\operatorname{diag}(0,1,0,0)$ and $R=$ 0 are used. Suppose, for the real system, $\beta(k)=1+$ $0.2 e^{-0.05 k} \cos (k)$. In the example of [13], the control horizon
$N$ cannot exceed 2 because of the sampling time of $0.1 \mathrm{~s}$. However, in this paper, we can select the initial control horizon $N$, larger than 2, without regard to the sampling time $T_{s}$. Figure 3 shows the comparison between the algorithm [13] with $N=2$ and the presented algorithm with $N=5$ and $N^{*}=1$. As a result, we can see that the proposed algorithm not only is useful for enlarging the stabilization set but also improves the control performance.

\section{Concluding Remarks}

In this paper, we presented an efficient MPC algorithm for uncertain time-varying systems with input constraints. The proposed algorithm was organized as two procedures: offline and on-line. In one procedure, we introduced an offline region-dependent MPC algorithm to enlarge the size of the stabilization set without regard to computational burdens. The off-line control scheme steers the state of systems into an invariant ellipsoid target set. In the other procedure, we proposed two on-line stabilizing MPC algorithms differentiated into $N^{*} \geq 1$ and $N^{*}=0$, which achieve local optimality within the neighborhood of the equilibrium point.

\section{Competing Interests}

The author declares that there is no competing interests regarding the publication of this paper. 


\section{Acknowledgments}

This work was supported by the 2016 Research Fund of University of Ulsan.

\section{References}

[1] S. C. Jeong and P. Park, "Constrained MPC algorithm for uncertain time-varying systems with state-delay," IEEE Transactions on Automatic Control, vol. 50, no. 2, pp. 257-263, 2005.

[2] A. G. S. Ottoni, R. H. C. Takahashi, and G. V. Raffo, "Stability constraints for robust model predictive control," Mathematical Problems in Engineering, vol. 2015, Article ID 870189, 11 pages, 2015.

[3] W.-W. Qin, G. Liu, L.-X. Wang, and Z.-Q. Zheng, "Memory state feedback RMPC for multiple time-delayed uncertain linear systems with input constraints," Mathematical Problems in Engineering, vol. 2014, Article ID 409863, 9 pages, 2014.

[4] J. B. Rawlings and D. Q. Mayne, Model Predictive Control: Theory and Design, Nob Hill, Madison, Wis, USA, 2009.

[5] P. Park and S. C. Jeong, "Constrained RHC for LPV systems with bounded rates of parameter variations," Automatica, vol. 40, no. 5, pp. 865-872, 2004.

[6] S. H. Kim, C. H. Lee, and P.-G. Park, " $H_{\infty}$ state-feedback control for fuzzy systems with input saturation via fuzzy weightingdependent Lyapunov functions," Computers \& Mathematics with Applications, vol. 57, no. 6, pp. 981-990, 2009.

[7] S. W. Yun, S. H. Kim, and J. Y. Park, "Asymptotic stabilization of continuous-time linear systems with input and state quantizations," Mathematical Problems in Engineering, vol. 2014, Article ID 947164, 6 pages, 2014.

[8] M. V. Kothare, V. Balakrishnan, and M. Morari, "Robust constrained model predictive control using linear matrix inequalities," Automatica, vol. 32, no. 10, pp. 1361-1379, 1996.

[9] Y. I. Lee and B. Kouvaritakis, "Stabilizable regions of receding horizon predictive control with input constraints," Systems \& Control Letters, vol. 38, no. 1, pp. 13-20, 1999.

[10] Y. I. Lee and B. Kouvaritakis, "A linear programming approach to constrained robust predictive control," IEEE Transactions on Automatic Control, vol. 45, no. 9, pp. 1765-1770, 2000.

[11] A. C. Brooms, B. Kouvaritakis, and Y. I. Lee, "Constrained MPC for uncertain linear systems with ellipsoidal target sets," Systems \& Control Letters, vol. 44, no. 3, pp. 157-166, 2001.

[12] F. A. Cuzzola, J. C. Geromel, and M. Morari, "An improved approach for constrained robust model predictive control," Automatica, vol. 38, no. 8, pp. 1183-1189, 2002.

[13] Z. Wan and M. V. Kothare, "Efficient robust constrained model predictive control with a time varying terminal constraint set," Systems \& Control Letters, vol. 48, no. 5, pp. 375-383, 2003.

[14] S. H. Kim, " $H_{\infty}$ output-feedback LPV control for systems with input saturation," International Journal of Control, Automation and Systems, vol. 10, no. 6, pp. 1267-1272, 2012.

[15] A. Casavola, M. Giannelli, and E. Mosca, "Min-max predictive control strategies for input-saturated polytopic uncertain systems," Automatica, vol. 36, no. 1, pp. 125-133, 2000.

[16] B. Kouvaritakis, J. A. Rossiter, and J. Schuurmans, "Efficient robust predictive control," IEEE Transactions on Automatic Control, vol. 45, no. 8, pp. 1545-1549, 2000.

[17] Y. I. Lee and B. Kouvaritakis, "Robust receding horizon predictive control for systems with uncertain dynamics and input saturation," Automatica, vol. 36, no. 10, pp. 1497-1504, 2000.
[18] MathWorks, "Robust control toolbox," http://kr.mathworks .com/help/robust/lmis. 


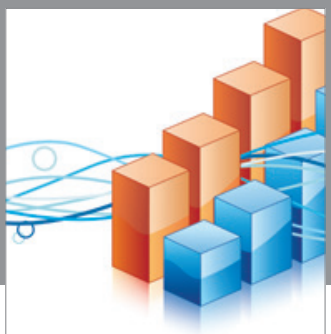

Advances in

Operations Research

vatem alat4

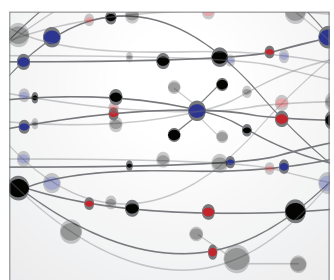

\section{The Scientific} World Journal
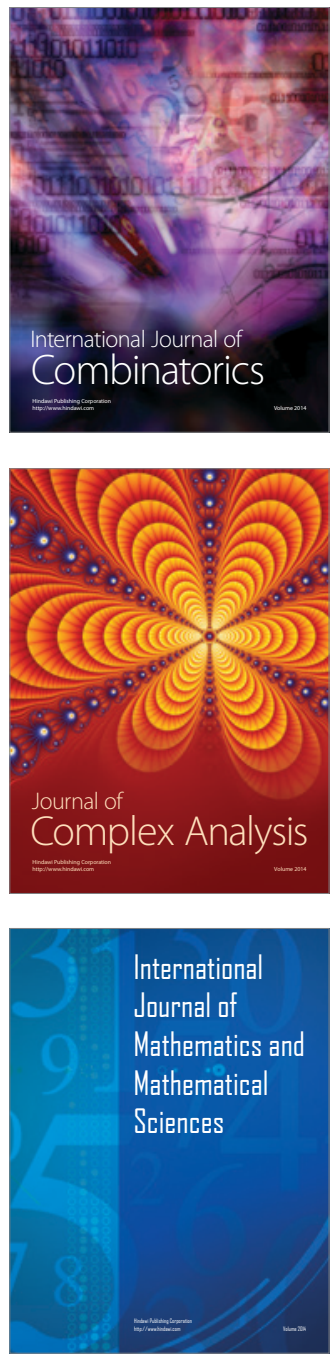
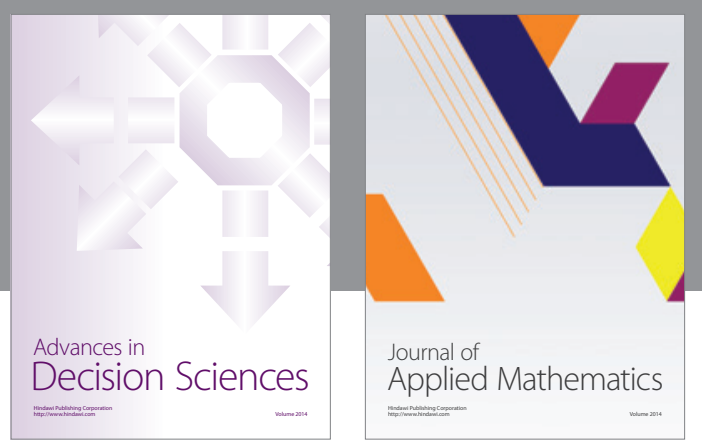

Algebra

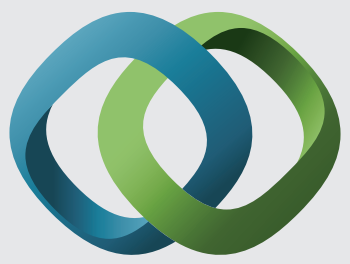

\section{Hindawi}

Submit your manuscripts at

http://www.hindawi.com
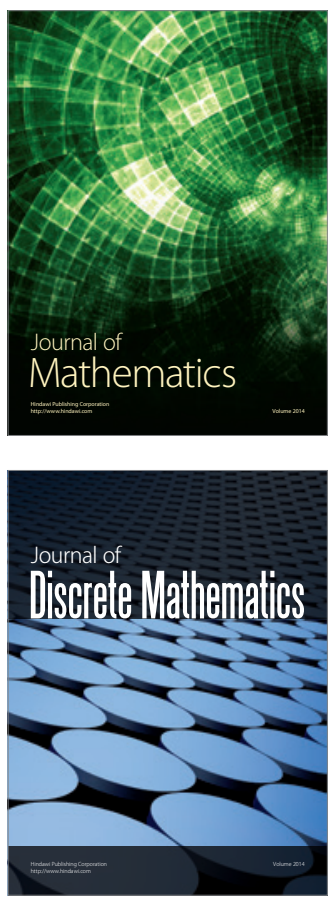

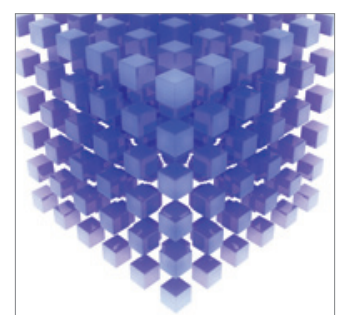

Mathematical Problems in Engineering
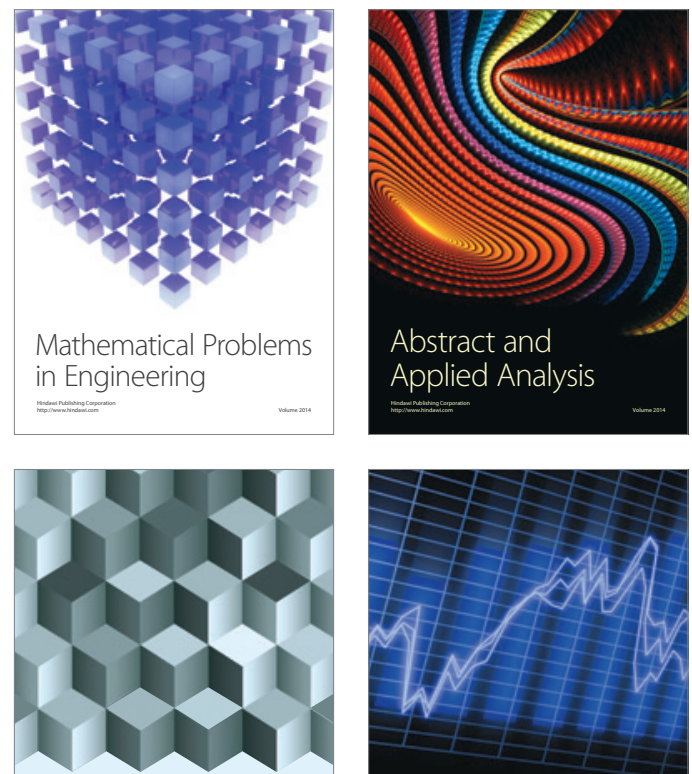

Journal of

Function Spaces

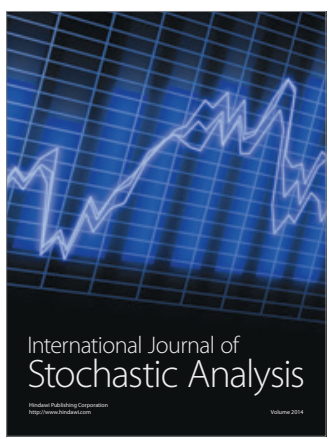

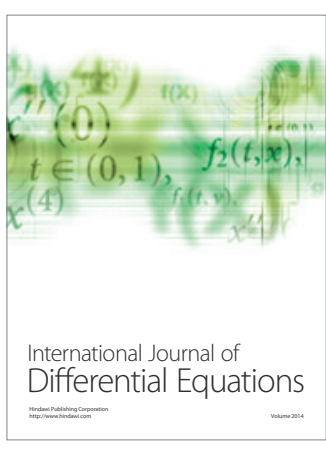
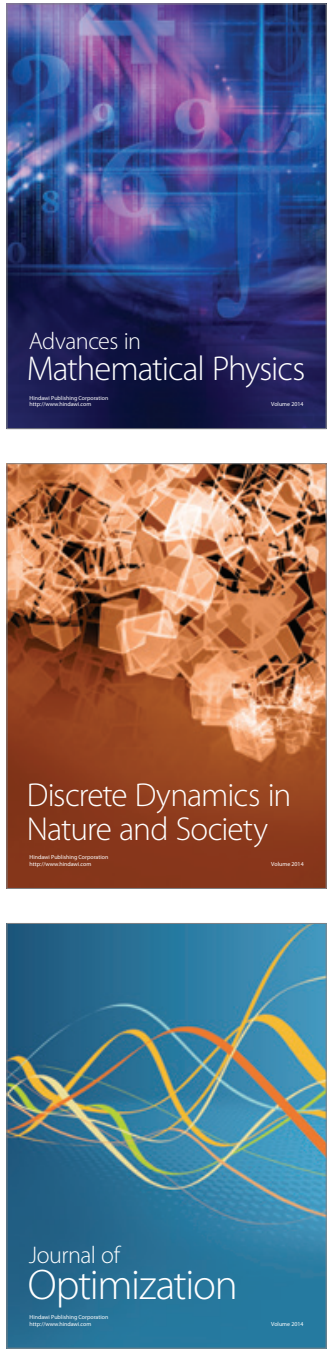